\title{
Madhubani Painting Motifs: A Source of Design Inspiration for Traditional to Contemporary Designs
}

\author{
Pooja Rani, Saroj Yadav*, Neelam M. Rose and Vandana Bhandari \\ Department of Textile and Apparel Designing, I.C. College of Home Science, CCS Haryana \\ Agriculture University, Hisar-125004(Haryana), India \\ *Corresponding author
}

\section{A B S T R A C T}

\begin{tabular}{|l|}
\hline K e y w o r d s \\
Motif, Design, \\
$\begin{array}{l}\text { Madhubani } \\
\text { painting, } \\
\text { CorelDRAW }\end{array}$ \\
\hline Article Info \\
\hline $\begin{array}{l}\text { Accepted: } \\
10 \text { October } 2019 \\
\text { Available Online: } \\
10 \text { November } 2019\end{array}$ \\
\hline
\end{tabular}

\section{Introduction}

India is a territory of diversity, extending from Himalaya in North to Kanyakumari in South, from Gujarat in West to Arunachal Pradesh in East. Each state of India has its uniqueness in terms of residing communities and their cultural aspects like costumes, traditions, rituals, attitudes, beliefs, language, arts and crafts. Cultural and traditional diversity make India multicultural nation where culture and people are very closely related with each other. The arts and crafts reflect rich heritage of India through beautiful designs inspired from monuments, architecture, religion, nature, etc. Arts and crafts define the cultural layers of a particular place and community. Every corner of India reflects definite 
specialties and uniqueness, showing the character of the area and its people (Bhandari, 2017).

Indian paintings offers a beautiful record of centuries, by reflecting religious beliefs, political event and social customs and provide an aesthetic continuum that extends from early civilization to present day. Indian paintings have evolved over the years to become a fusion of various cultures and traditions. In India, there is great variety of folk and tribal paintings such as Patachitra paintings from Odisha, Phad and Pichhvai Paintings of Rajasthan, Kalamkari of Andhra Pradesh, Warli Paintings of Maharashtra, Nirmal Paintings of Andhra Pradesh, Aipan of Uttarakhand, Pithoro paintings of Gujarat, Gond and Mandana Paintings of Madhya Pradesh, Kalighat paintings of Calcutta, Madhubani from Bihar and many more forms.

Madhubani paintings, one of the most celebrated work of art from Bihar are accomplished by generations of skilled artists. Being vibrant and symbolic at the same time, these successfully depict all aspects of tribal life. In other words, these are the representative of folk art in visual form. Madhubani painting is also known as Mithila painting due to practicing in Mithila region of Bihar and Nepal.

The Indian arts and crafts can play an important role in fashion world. As now a day's fashion trends are fragile and consumer constantly demand innovative design and change over existing fashion trends. Designers and manufacturers constantly face the challenges of providing new designs to suit the rapidly growing fashion consciousness in the market. It is important to keep innovative but at the same time taking inspiration from the rich treasure of our traditional textiles and crafts will help to keep them alive, relevant and marketable (Vaishnav, 2015). Thus the present study was conducted to adapt traditional motifs of madhubani painting for development of designs for contemporary applications.

\section{Materials and Methods}

The present study was conducted in the Department of Textile and Apparel Designing, I.C. College of Home Science, CCS Haryana Agricultural University, Hisar.

\section{Collection of Motifs}

A total of two hundred sixty four traditional Madhubani motifs were collected from various secondary sources like books, journals, encyclopedia, magazines, web sites etc. All the collected motifs were analyzed critically and on the basis of their suitability to develop innovative designs, 120 motifs were screened and were classified as floral \& foliage, geometrical, birds \& animals and religious motifs.

\section{Selection of Motifs}

A preferential choice index was prepared, to obtain the preferences regarding selected motifs on three point rating scale i.e. highly preferred, preferred and least preferred. Weighted Mean scores were calculated for all the motifs according to the preferences obtained. On the basis of weighted mean scores, ranks were assigned to the motifs. Total sixteen motifs i.e. four motifs from each category were selected to develop designs for varied application.

\section{Development of designs}

The selected sixteen motifs were used for creation of innovative contemporary designs. The simulation of motifs was done with the help of CorelDRAW X5 using various software tools for enlargement/reduction of size, transformation, modification, rotation, editing until an aesthetically pleasing and 
required arrangement of motifs was obtained. A total of thirty designs were developed from the selected sixteen motifs using two to four motifs as per their compatibility and harmony.

\section{Selection of designs}

A preferential choice index was used for selection of designs and individual score of each created design was calculated as weighted mean scores. Ranks were assigned according to their weighted mean scores and the top preferred five designs were selected for contemporary application.

\section{Results and Discussion}

\section{Collection of Motifs}

Total one hundred twenty traditional motifs of Madhubani painting comprising of floral \& foliage, geometrical, birds \& animals and religious motifs etc. were collected personally through photographs from the market of Hisar city and secondary sources of data like books, journals and internet. The collected motifs were refined/ recreated in Corel DRAW X5 to get the required intricacy and fineness (Plate I).

\section{Selection of motifs}

The collected motifs were shown to thirty experts for selections of motifs for design development. The results obtained are presented in Table 1 to 4 and selected motifs are shown in Plate 2.

The data pertaining to preferences of experts for selection of motifs depicted in table 1 reveal that motif number 29 was ranked $I^{\text {st }}$ (WMS 2.76) followed by motif number 30 (WMS 2.60) ranked II $^{\text {nd }}$, motif number 11(WMS 2.56) ranked $\mathrm{III}^{\text {rd }}$ and motif number 8(WMS 2.46) ranked $\mathrm{IV}^{\text {th }}$. The data presented in Table 2 depicts that as per the preferences of experts, motif number 8 scored highest weighted mean score i.e. 2.80, ranked I in geometrical motifs category followed by motif number 26 (WMS 2.73) ranked II, motif number 2 (WMS 2.66) ranked III and motif number 15 (WMS 2.56) ranked IV.

Table.1 Preferential choices for floral and foliage motifs

\begin{tabular}{|c|c|c|c|c|c|}
\hline \multicolumn{7}{|c|}{$(\mathrm{n}=30)$} \\
\hline Motif No. & WMS & Rank & Motif No. & WMS & Rank \\
\hline $\mathbf{1}$ & 2.03 & XV & 16 & $\mathbf{2 . 4 6}$ & IV \\
\hline $\mathbf{2}$ & 2.13 & XII & 17 & 2.4 & VI \\
\hline $\mathbf{3}$ & 1.83 & XXIV & 18 & 2.36 & VII \\
\hline $\mathbf{4}$ & 2.23 & X & 19 & $\mathbf{2 . 5 6}$ & III \\
\hline $\mathbf{5}$ & 2.1 & XIII & 20 & 1.76 & XXV \\
\hline $\mathbf{6}$ & 2.26 & IX & 21 & 1.96 & XX \\
\hline $\mathbf{7}$ & 1.6 & XXVIII & 22 & 1.86 & XXIII \\
\hline $\mathbf{8}$ & 1.36 & XXX & 23 & 1.96 & XX \\
\hline $\mathbf{9}$ & 1.66 & XXVII & 24 & 2.2 & XI \\
\hline $\mathbf{1 0}$ & 2.1 & XIII & 25 & 2 & XVIII \\
\hline $\mathbf{1 1}$ & 2 & XVIII & 26 & $\mathbf{2 . 4 3}$ & V \\
\hline $\mathbf{1 2}$ & 2.03 & XV & 27 & 1.7 & XXVI \\
\hline $\mathbf{1 3}$ & 1.93 & XXII & 28 & 2.02 & XVII \\
\hline $\mathbf{1 4}$ & 1.6 & XIX & 29 & $\mathbf{2 . 7 6}$ & I \\
\hline $\mathbf{1 5}$ & 2.3 & VIII & 30 & $\mathbf{2 . 6}$ & II \\
\hline
\end{tabular}

WMS- Weighted Mean Score 
Table.2 Preferential choices for geometrical motifs

\begin{tabular}{|c|c|c|c|c|c|}
\hline \multicolumn{7}{|c|}{ Geometrical Motifs } & \multicolumn{1}{c|}{} \\
\hline Motif No. & WMS & Rank & Motif No. & WMS & Rank \\
\hline $\mathbf{1}$ & $\mathbf{1 . 8 6}$ & $\mathbf{V}$ & 9 & 2 & XXIII \\
\hline $\mathbf{2}$ & $\mathbf{2 . 2 6}$ & III & 10 & 2.56 & VIII \\
\hline $\mathbf{3}$ & 2.2 & XIX & 11 & 2.36 & XIII \\
\hline $\mathbf{4}$ & 1.7 & XXIX & 12 & 2.54 & IX \\
\hline $\mathbf{5}$ & 1.86 & XXVI & 13 & 2.36 & XIII \\
\hline $\mathbf{6}$ & 2.33 & XV & 14 & 2.66 & VII \\
\hline $\mathbf{7}$ & 2.42 & X & 15 & $\mathbf{2 . 2}$ & IV \\
\hline $\mathbf{8}$ & $\mathbf{2 . 8}$ & I & 16 & 2.3 & XII \\
\hline $\mathbf{1 7}$ & 2.22 & XVIII & 24 & 2.13 & XX \\
\hline $\mathbf{1 8}$ & 1.93 & XXIV & 25 & 2.73 & VI \\
\hline $\mathbf{1 9}$ & 2.33 & XV & 26 & $\mathbf{2 . 5}$ & II \\
\hline $\mathbf{2 0}$ & 1.86 & XXVI & 27 & 2.4 & XI \\
\hline $\mathbf{2 1}$ & 2.23 & XVII & 28 & 1.83 & XXVIII \\
\hline $\mathbf{2 2}$ & 1.93 & XXIV & 29 & 1.53 & XXX \\
\hline $\mathbf{2 3}$ & 2.1 & XXII & 30 & 2.13 & XX \\
\hline
\end{tabular}

WMS- Weighted Mean Score

Table.3 Preferential choices for animal and bird motifs

\begin{tabular}{|c|c|c|c|c|c|}
\hline \multicolumn{7}{|c|}{$(\mathrm{n}=30)$} \\
\hline Motif No. & WMS & Rank & Motif No. & WMS & Rank \\
\hline $\mathbf{1}$ & $\mathbf{2 . 8}$ & I & 6 & 2.5 & VI \\
\hline $\mathbf{2}$ & 1.63 & XXVII & 7 & 2.16 & IX \\
\hline $\mathbf{3}$ & 1.86 & XXII & 8 & 1.7 & XXV \\
\hline $\mathbf{4}$ & 2.06 & XVI & 9 & 2.05 & XVII \\
\hline $\mathbf{5}$ & 2.03 & XIX & 10 & 1.66 & XXVI \\
\hline $\mathbf{1 1}$ & 2.43 & VII & 19 & 1.86 & XXII \\
\hline $\mathbf{1 2}$ & 2.2 & IX & 20 & 2.04 & XVIII \\
\hline $\mathbf{1 3}$ & 1.83 & XXIV & 21 & 2.43 & VII \\
\hline $\mathbf{1 4}$ & 1.96 & XXI & 22 & 2.13 & XIII \\
\hline $\mathbf{1 5}$ & $\mathbf{1 . 8 3}$ & IV & 23 & 2.03 & XX \\
\hline $\mathbf{1 6}$ & $\mathbf{2 . 1 6}$ & III & 24 & $\mathbf{2 . 4 3}$ & II \\
\hline $\mathbf{1 7}$ & 2.16 & XI & 25 & 2.1 & XIV \\
\hline $\mathbf{1 8}$ & 1.56 & XXVIII & 26 & 2.1 & XIV \\
\hline $\mathbf{2 7}$ & $\mathbf{1 . 6 3}$ & V & 29 & 1.5 & XXX \\
\hline $\mathbf{2 8}$ & 2.16 & XI & 30 & 1.56 & XXVIII \\
\hline
\end{tabular}

WMS- Weighted Mean Score 
Table.4 Preferential choices for religious motifs

\begin{tabular}{|c|c|c|c|c|c|}
\hline \multicolumn{7}{|c|}{ Religious Motifs } \\
\hline Motif No. & WMS & Rank & Motif No. & WMS & Rank \\
\hline $\mathbf{1}$ & 2.16 & IX & 16 & 2.13 & XI \\
\hline $\mathbf{2}$ & $\mathbf{2 . 2 6}$ & I & 17 & 2.1 & XIII \\
\hline $\mathbf{3}$ & 2.16 & IX & 18 & 2.2 & VII \\
\hline $\mathbf{4}$ & 2.03 & XII & 19 & 2.13 & XI \\
\hline $\mathbf{5}$ & 1.9 & XXI & 20 & $\mathbf{2 . 7 6}$ & III \\
\hline $\mathbf{6}$ & 1.96 & XX & 21 & 2.26 & VI \\
\hline $\mathbf{7}$ & 1.55 & XXX & 22 & 2.03 & XVII \\
\hline $\mathbf{8}$ & 2.03 & XIX & 23 & 1.9 & XXI \\
\hline $\mathbf{9}$ & 1.84 & XXVI & 24 & 1.6 & XXIX \\
\hline $\mathbf{1 0}$ & 1.86 & XXIV & 25 & 1.7 & XXVIII \\
\hline $\mathbf{1 1}$ & 2.06 & XVI & 26 & $\mathbf{2 . 3 3}$ & V \\
\hline $\mathbf{1 2}$ & 2.2 & VII & 27 & 1.89 & XXIII \\
\hline $\mathbf{1 3}$ & 1.82 & XXVII & 28 & $\mathbf{2 . 3}$ & IV \\
\hline $\mathbf{1 4}$ & 2 & XIV & 29 & 2 & XIV \\
\hline $\mathbf{1 5}$ & 1.86 & XXIV & 30 & $\mathbf{1 . 8 6}$ & II \\
\hline
\end{tabular}

WMS- Weighted Mean Score

Table.5 Preferential choices for developed designs

\begin{tabular}{|c|c|c|c|c|c|}
\hline \multicolumn{7}{|c|}{$(\mathrm{n}=30)$} \\
\hline Design No. & WMS & Rank & Design No. & WMS & Rank \\
\hline $\mathbf{1}$ & 1.81 & XXVIII & 16 & 1.83 & XXVII \\
\hline $\mathbf{2}$ & 1.90 & XXIV & 17 & 2.19 & XIII \\
\hline $\mathbf{3}$ & 1.95 & XXII & 18 & 2.11 & XVIII \\
\hline $\mathbf{4}$ & 2.14 & XV & 19 & 2.03 & XXI \\
\hline $\mathbf{5}$ & 2.12 & XVII & $\mathbf{2 0}$ & $\mathbf{2 . 6 5}$ & II \\
\hline $\mathbf{6}$ & 1.72 & XXX & 21 & 2.25 & IX \\
\hline $\mathbf{7}$ & 2.23 & XI & $\mathbf{2 2}$ & $\mathbf{2 . 4 4}$ & IV \\
\hline $\mathbf{8}$ & 2.18 & XIV & $\mathbf{2 3}$ & $\mathbf{2 . 3 3}$ & V \\
\hline $\mathbf{9}$ & 2.29 & VII & 24 & 1.81 & XXVIII \\
\hline $\mathbf{1 0}$ & 2.23 & XI & 25 & 1.87 & XXVI \\
\hline $\mathbf{1 1}$ & 2.09 & XIX & 26 & 2.28 & VIII \\
\hline $\mathbf{1 2}$ & 1.88 & XXV & 27 & 2.13 & XVI \\
\hline $\mathbf{1 3}$ & 2.32 & VI & 28 & 2.24 & X \\
\hline $\mathbf{1 4}$ & $\mathbf{2 . 6 9}$ & I & $\mathbf{2 9}$ & $\mathbf{2 . 6 0}$ & III \\
\hline $\mathbf{1 5}$ & 2.07 & XX & 30 & 1.93 & XXIII \\
\hline
\end{tabular}

WMS- Weighted Mean Score 
Plate.1 Collected Madhubani Motifs

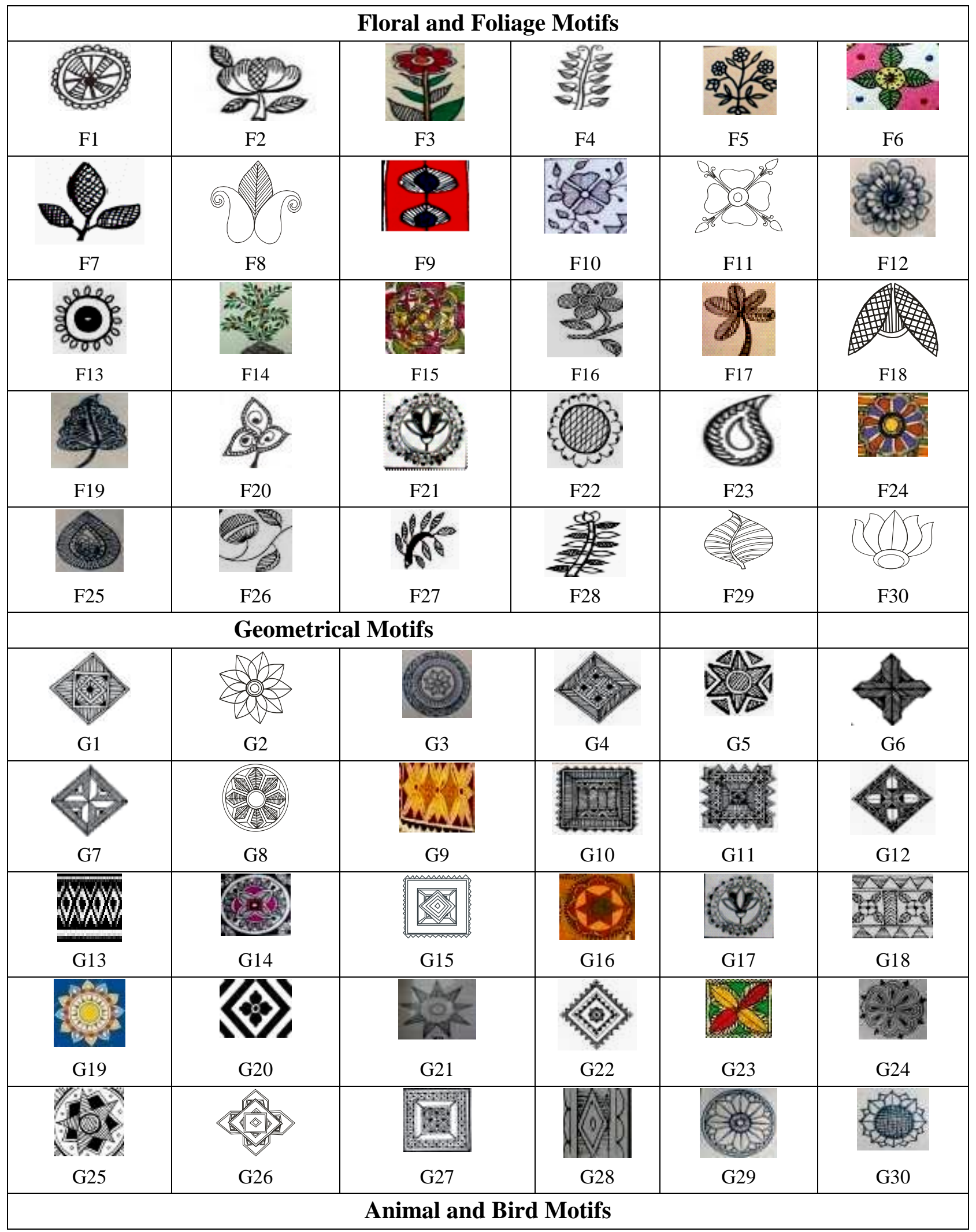




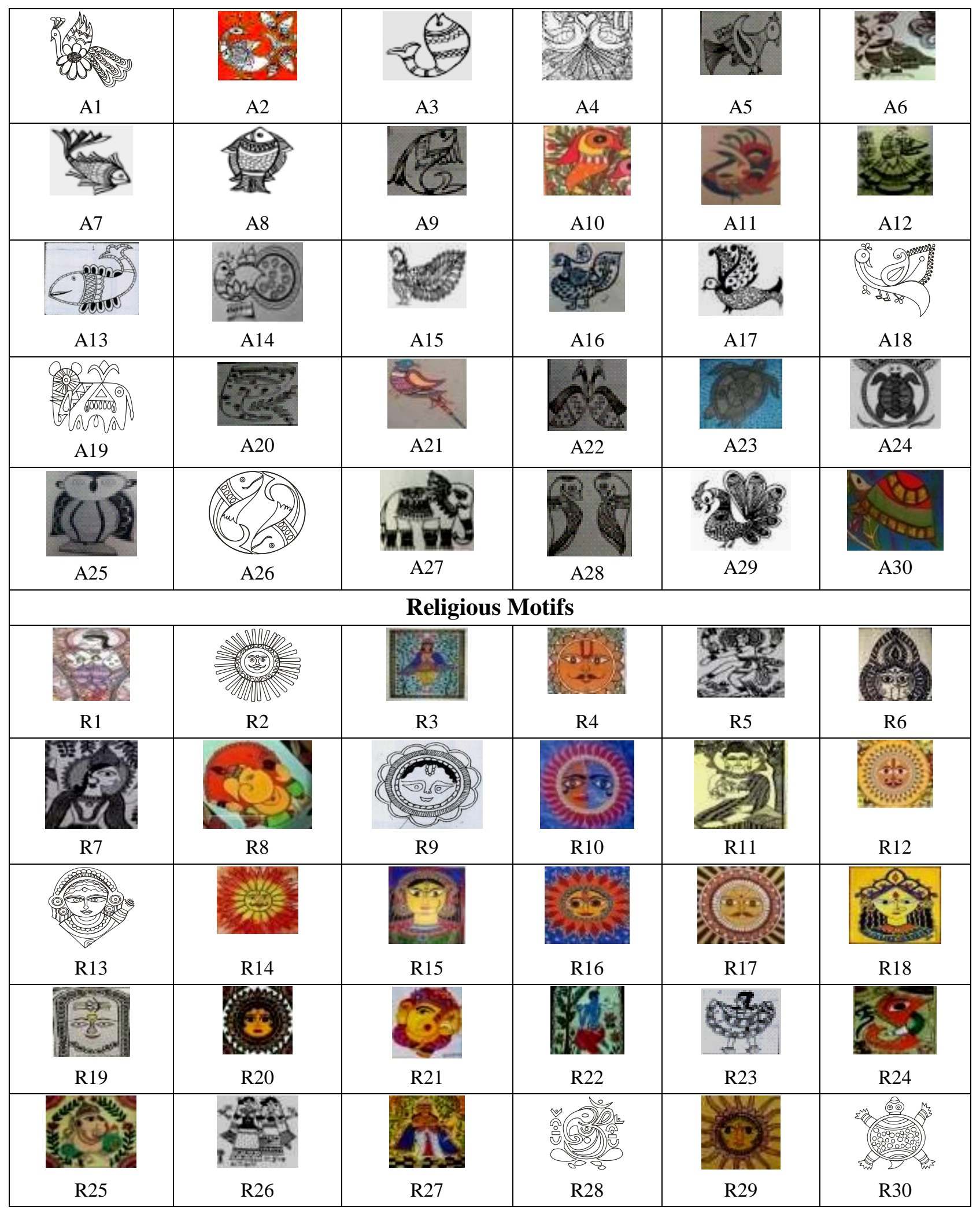


Plate.2 Selected Motifs for Design Development

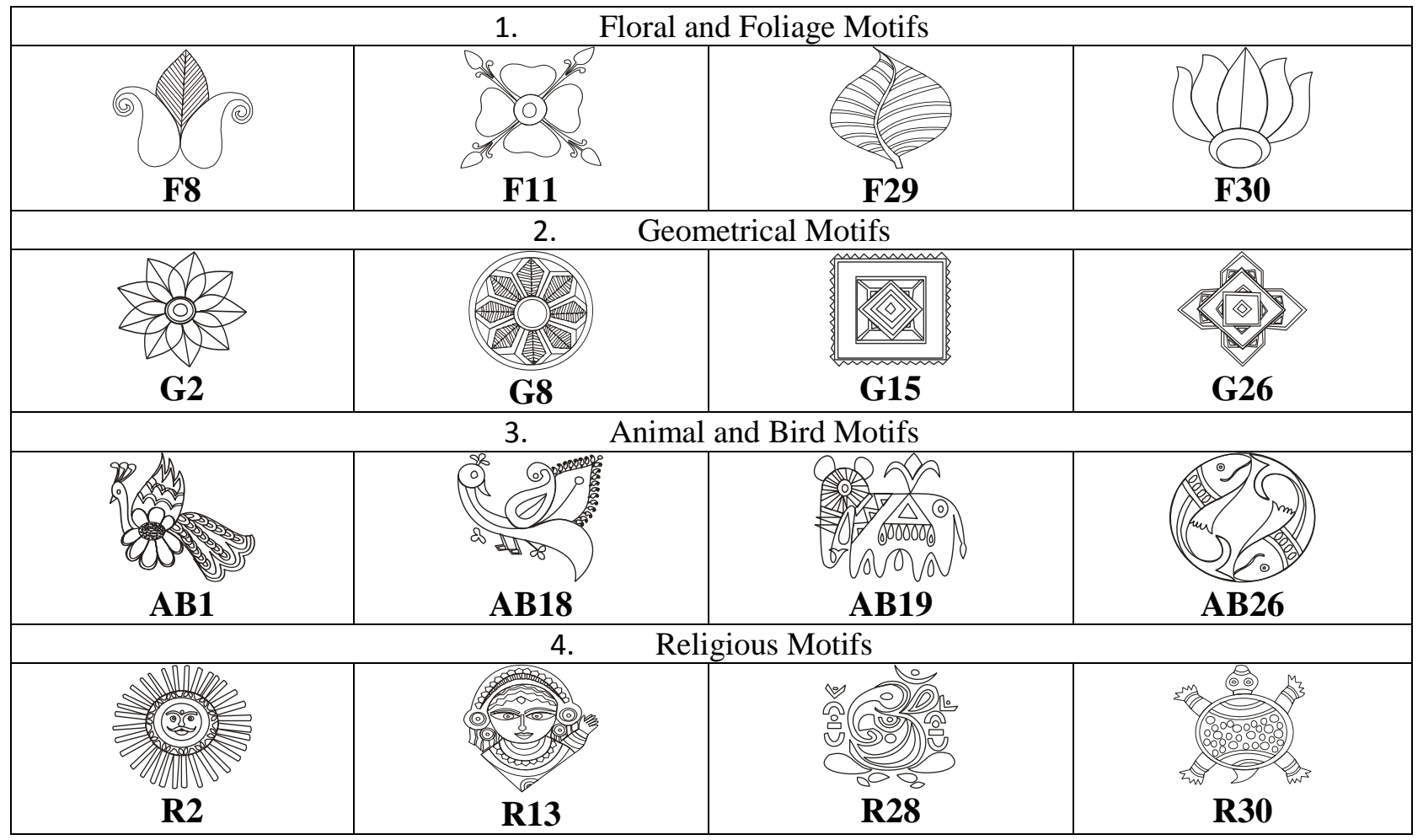

Plate.3 Developed Designs

\begin{tabular}{|c|c|c|c|c|}
\hline$\frac{(\mathrm{S})}{1(\mathrm{G} 2 \mathrm{R} 1,4)}$ & 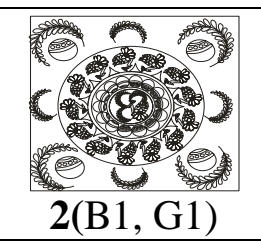 & 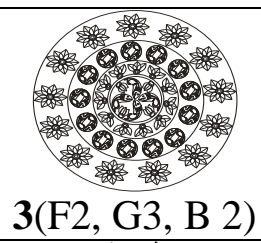 & $\begin{array}{c}0 \\
4(\mathrm{G} 1,2) \\
0\end{array}$ & $\begin{array}{c}3 \\
5(\mathrm{~F} 1,3, \mathrm{G} 1)\end{array}$ \\
\hline $\begin{array}{l}6=0 \\
6(F 1,2)\end{array}$ & 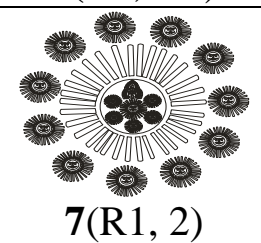 & 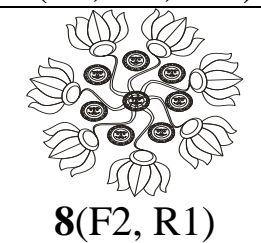 & $\mathbf{9}(\mathrm{F} 1,3)$ & $\begin{array}{c}\text { (1) } \\
10(\mathrm{~F} 1, \mathrm{R} 4, \mathrm{G} 3)\end{array}$ \\
\hline 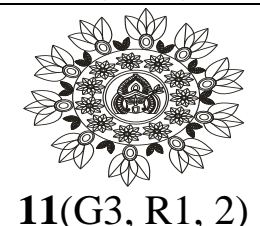 & $12(\mathrm{G} 2, \mathrm{~B} 5)$ & 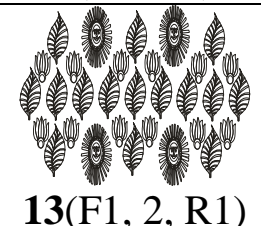 & $14(R 1, G 3)$ & $\mathbf{1 5}(\mathrm{A} 2, \mathrm{R} 1)$ \\
\hline $\begin{array}{c}169 \\
16(\mathrm{R} 1,3)\end{array}$ & 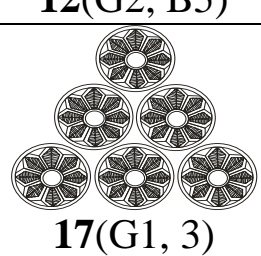 & 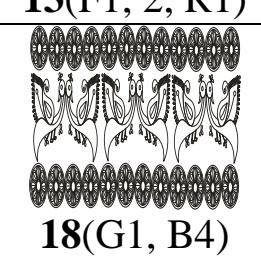 & 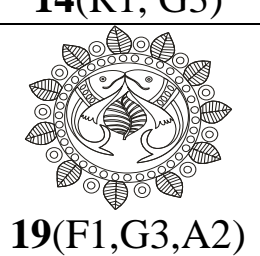 & 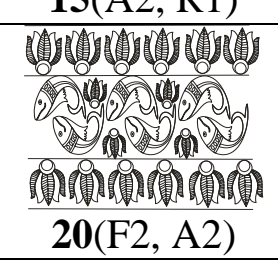 \\
\hline
\end{tabular}




\begin{tabular}{|c|c|c|c|c|}
\hline 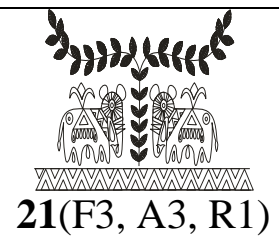 & 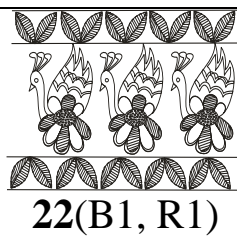 & 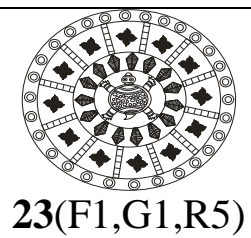 & $\begin{array}{l}24(\mathrm{~F} 3, \mathrm{G} 3) \\
20\end{array}$ & 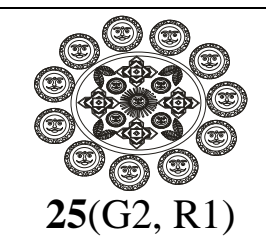 \\
\hline $\begin{array}{c}1 \\
\mathbf{2 6}(\mathrm{F} 2, \mathrm{R} 1)\end{array}$ & 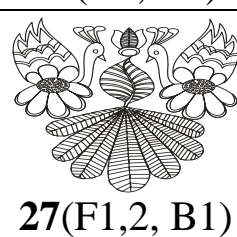 & $\begin{array}{c}1 \\
-1 \\
-1.5\end{array}$ & 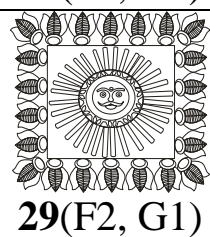 & $\begin{array}{c}\text { (1) } \\
30(\mathrm{~F} 2, \mathrm{G} 2, \mathrm{~B} 1)\end{array}$ \\
\hline
\end{tabular}

Plate.4 Selected Designs

(1)

The data regarding preferences of experts for animal \& birds motifs category presented in Table 3 narrate that motif number 1 scored highest weighted mean score i.e. 2.80, ranked
I followed by motif number 26 (WMS 2.66) ranked II, motif number 19 (WMS 2.60) ranked III and motif number 18 (WMS 2.56) ranked IV. 
The data pertaining to preferences of experts for religious motifs incorporated in Table 4 highlighted that motif number 2 scored highest weighted mean score i.e. 2.76, ranked I followed by motif number 13 (WMS 2.65) ranked II, motif number 28 (WMS 2.60) ranked III and motif number 30 (WMS 2.57) ranked IV.

It is thus inferred that four top preferred motifs from each category were selected for the development of designs were motif number 29, 30, 11 and 8 from floral and foliage, motif

\section{Selection of designs}

All the developed designs were visually evaluated by thirty experts and their preferences were obtained for selection of designs for contemporary applications (Table 5).

The data in Table 5 shows the preferences of experts for developed designs. As per the experts' preferences design number 14 was preferred the most and ranked $\mathrm{I}^{\text {st }}$ scoring highest weighted mean score i.e. 2.69, followed by design number 20 (WMS 2.65) at rank II, design number 29 (WMS 2.60) at rank III, design number 22 (WMS 2.44) at rank IV and design number 23(WMS 2.33) at rank V. Sharma et al., 2016 also developed contemporary designs using traditional motifs. Top preferred designs were used for creation of design placements on sari. Gupta and Joseph, 2015 developed designs for table cloth using Auto CAD software. Forty Greek motifs in eight different categories i.e. animal, human figure, pottery, foliage, stylized, architectural, geometrical and stylized floral fauna were used to develop twenty designs. Devi et al., 2017 reported that motifs play an important role in designing. Each motif, like the fabric itself has an origin, evolution and variety in shape and presentation. In designing, a weaver, embroiderer, dyer or printer creates number 8, 26, 2 and 15 from geometrical, motif number 1, 26, 19 and 18 from bird and animal and motif number 2, 13, 28 and 30 from religious.

\section{Development of designs}

The selected sixteen motifs were combined in systematic manner using CorelDRAW X5 software for design development for stoles. Total thirty designs were developed using selected motifs and presented in Plate-3.

dreams of beauty using motifs familiar through culture, religion, environment and history on textiles. Development in any field is essential and continuing process so in the field of textile designing.

It is concluded from the present findings that traditional Madhubani motifs can be adapted through CorelDRAW software for development of innovative designs for varied application. Developed designs can be applied to textile products through different techniques of surface enrichment like digital embroidery, hand embroidery, printing, painting and combination of printing and embroidery.

The developed designs can be given to entrepreneurs for mass production to help them in establishing small scale enterprises for developing a wide range of textile items. Further, this effort will help to preserve the traditional art form of Bihar state.

\section{References}

Bhandari V. 2017. Development of borders through extra yarn figuring using adapted Aipan designs for application on apparel and accessories. M.Sc. Thesis, G. B. Pant University of Agriculture and Technology, Pantnagar, Uttarakhand. 
Devi S. Punia P. Pruthi N. and Sisodia N. 2017. Development of Kantha Embroidery Motifs to Designs: Traditional to Contemporary. International Journal of Current Microbiology and Applied Sciences, 6(7): 4479-4488.

Gupta V and Joseph R. 2015. Development of designs for table cloth through CAD software. Asian Journal of Home Science, 10(1): 60-64.
Sharma, A., Singh S. S. J., and Rose. N. M. 2016. Development of motifs: Traditional to contemporary for sarees. Research Journal of Recent Sciences, 5(7): 44-46.

Vaishnav S. 2015. Development of customized software on Warli folk art of Maharashtra for value addition. Ph.D. Thesis, Maharana Pratap University of Agriculture and Technology, Udaipur, Rajasthan.

\section{How to cite this article:}

Pooja Rani, Saroj Yadav, Neelam M. Rose and Vandana Bhandari. 2019. Madhubani Painting Motifs: A Source of Design Inspiration for Traditional to Contemporary Designs. Int.J.Curr.Microbiol.App.Sci. 8(11): 863-873. doi: https://doi.org/10.20546/ijcmas.2019.811.102 